\title{
Biological Implications of a Discrete Mathematical Model for Collagen Deposition and Alignment in Dermal Wound Repair
}

\author{
J. C. Dallon \\ dallon@math.byu.edu \\ J. A. Sherratt \\ P. K. Maini \\ M. W. Ferguson
}

Follow this and additional works at: https://scholarsarchive.byu.edu/facpub

Part of the Mathematics Commons

\section{Original Publication Citation}

J.C. Dallon, J.A. Sherratt, P.K. Maini and M.W. Ferguson: Biological Implications of a Discrete Mathematical Model for Collagen Deposition and Alignment in Dermal Wound Repair. IMA Journal of Mathematics Applied in Medicine and Biology 17: 379-393 (2).

\section{BYU ScholarsArchive Citation}

Dallon, J. C.; Sherratt, J. A.; Maini, P. K.; and Ferguson, M. W., "Biological Implications of a Discrete Mathematical Model for Collagen Deposition and Alignment in Dermal Wound Repair" (2000). Faculty Publications. 1102.

https://scholarsarchive.byu.edu/facpub/1102 accepted for inclusion in Faculty Publications by an authorized administrator of BYU ScholarsArchive. For more information, please contact ellen_amatangelo@byu.edu. 


\title{
Biological Implications of a Discrete Mathematical Model for Collagen Deposition and Alignment in Dermal Wound Repair
}

John Dallon ${ }^{1}$, Jonathan Sherratt ${ }^{2}$, Philip Maini ${ }^{3}$ and Mark Ferguson ${ }^{4}$

\author{
${ }^{1}$ Department of Mathematics \\ Brigham Young University \\ Provo, UT 84602-6539 U.S.A. \\ ${ }^{2}$ Department of Mathematics \\ Heriot-Watt University \\ Edinburgh, EH14 4AS U.K. \\ ${ }^{3}$ Centre for Mathematical Biology \\ Mathematical Institute \\ University of Oxford \\ 24-29 St Giles' \\ Oxford OX1 3LB, U.K. \\ ${ }^{4}$ School of Biological Sciences \\ University of Manchester \\ Oxford Road \\ Manchester M13 9PT, U.K.
}

August 15, 2000

RUNNING TITLE: A Model of Collagen Deposition in Wound Repair 


\begin{abstract}
We develop a novel mathematical model for collagen deposition and alignment during dermal wound healing. We focus on the interactions between fibroblasts, modelled as discrete entities, and a continuous extracellular matrix composed of collagen and a fibrin based blood clot. There are four basic interactions assumed in the model: fibroblasts orient the collagen matrix, fibroblasts produce and degrade collagen and fibrin and the matrix directs the fibroblasts and determines the speed of the cells. Several factors which influence the alignment of collagen are examined and related to current anti-scarring therapies using transforming growth factor $\beta$. The most influential of these factors are cell speed and, more importantly for wound healing, the influx of fibroblasts from surrounding tissue.
\end{abstract}




\section{Introduction}

The healing of full-thickness skin wounds involves a complex sequence of overlapping events. One of the first is the coagulation cascade (Jennings \& Hunt, 1992) where fibrinogen, a soluble protein in the plasma, is converted to fibrin which polymerizes to form a blood clot. This clot forms a provisional matrix for the invasion of various cell types. Within twenty four to forty eight hours (Jennings \& Hunt, 1992; Forrest, 1983), fibroblasts start to infiltrate the wound and dissolve the fibrin clot, replacing it with a collagen matrix. This is the process which will be modelled in this paper. In the final phase of wound healing the composition of the extracellular matrix is changed over a period of months, again by dermal fibroblasts. Experiments showing the growth of scars in parallel with animal growth (Armstrong \& Ferguson, 1995) suggest a stable, selfreinforcing mechanism. Based on evidence such as this, the orientation of the initially deposited collagen is thought to be key in subsequent remodelling, setting a pattern which is then perpetuated. Thus, we focus on the initial collagen deposition. Because wound healing is a complex phenomenon with many interacting factors, mathematical modelling is an ideal tool by which this process can be understood.

Many mathematical models have been developed which investigate various aspects of wound healing. Modelling efforts for dermal repair started by studying wound contraction using a mechanochemical framework originally formulated for embryological pattern formation (Tranquillo \& Murray, 1992). This model was extended to include different cell types (Olsen et al., 1995) and a study of nonlinear mechanical properties (Tracqui et al., 1995). These models of wound contraction deal with wound healing at a later stage than that which we are considering. Our work is most closely related to previous models which focus on collagen composition (Dale et al., 1996) and structure (Olsen et al., 1999), but we emphasize the collagen alignment to a greater extent and we incorporate fibroblasts in a more biologically realistic manner than previous models. In our work the fibroblasts are discrete cells, thus we use discrete components in our modelling approach, a technique which is now being adopted by a number of researchers to model problems in areas related to wound healing, such as angiogenesis and tumour growth and invasion (Anderson \& Chaplain, 1998; Stott et al., 1999; Anderson et al., 2000).

There are fundamental differences between fetal and adult wound healing, resulting 
in different outcomes. In adults, the end product is a scar. This is tissue which is less functional, weaker and cosmetically undesirable. However fetal tissue is repaired with reduced or absent scarring (McCallion \& Ferguson, 1996) and the realization of this difference has generated a large volume of research activity into mechanisms underlying scar formation. The characteristics of scars mentioned previously are a result of altered structure and composition in the dermis. Scars typically have fewer blood vessels supplying the denser connective tissue, which is less elastic. The most significant difference between normal tissue and scar tissue seems to be the orientation of the fibrous matrix. In rodents, normal tissue has a reticular collagen pattern, whereas the collagen in scar tissue forms large parallel bundles at approximately right angles to the basement membrane (Whitby \& Ferguson, 1991). Human scar tissue is similar, with greater collagen density, larger fibres and more alignment than normal tissue, although the alignment is parallel to the skin (Ehrlich \& Krummel, 1996). By modelling the production of collagen during tissue regeneration, our aim is to understand how the fibroblasts orient the collagen matrix and thus form scars.

This paper is organized in the following manner. In the next section the model is described. In Section 3 the numerical results are presented by first exploring the effects of altering key parameters in the model, and the experimental predictions are discussed in Section 4. Finally, in Section 5 we conclude with a discussion of the model implications as related to antiscarring therapies and future work.

\section{The Model}

Our model focuses on the interaction between fibroblasts within the wound and the extracellular matrix surrounding them. This interaction divides naturally into two categories: the influence of the extracellular matrix on the fibroblasts and the influence of the fibroblasts on the extracellular matrix. After describing the general framework of the model, we consider these two interactions in turn.

Traditionally, models of wound healing have represented the evolving components as continuum density variables, using partial differential equations to model the interactions. The approach taken here is different in that the cells are represented as discrete objects. Previous authors have studied cellular automata models for fibroblast populations (Edelstein-Keshet \& Ermentrout, 1990), but our work is novel with its inclusion of 
cell and extracellular matrix interactions. Although a continuum approach makes the model more amenable to mathematical analysis, it is less realistic for the low fibroblast densities which are found in wound healing, where the discrete nature of the fibroblasts is important for the observed microscopic patterns of alignment. This key difference profoundly influences the formulation of the model, which consists of ordinary differential equations, with the influence of the fibroblasts being strictly local. The model keeps track of the individual paths of the fibroblasts, denoted by $\mathbf{f}^{i}$ for $i=1,2, \ldots, M$ where $M$ is the total number of cells, and has parameters representing cell properties. Cells can enter the wound region either by division or by influx at the boundary of the wound region, representing fibroblasts entering from surrounding tissue.

The representation of the extracellular matrix follows the traditional modelling route in that it is a continuous variable, with vector fields of collagen and fibrin (the blood clot) denoted by $\mathbf{c}(\mathbf{x})$ and $\mathbf{b}(\mathbf{x})$ respectively. The direction of these vectors represents the predominant direction of the protein fibres at the point, and their length corresponds to the density of protein (collagen or fibrin) which is scaled to be between 0 and 1.5 for the collagen and 0 and 1 for the fibrin. Thus $\|\mathbf{c}\|=1$ corresponds to the collagen density of normal skin and $\|\mathbf{b}\|=1$ represents the fibrin density of a typical blood clot. Since the extracellular matrix is composed of non-directional fibres, the orientation can be represented by one of two directions which differ by 180 degrees. This means that the collagen and fibrin are not represented uniquely, so that some care is required in model simulations.

Extracellular matrix regulation of fibroblast activity. Although several of the extracellular matrix-fibroblast interactions have been experimentally studied, this area remains poorly understood (Hay, 1991). This is partly because all interactions have not been found, but mainly because the processes involved interact in a complex manner with nonlinear feedback. Mathematical modelling is a powerful tool designed to address such complex feedback mechanisms. For simplicity we consider only two effects on the fibroblasts from the extracellular matrix both of which are experimentally well documented. First, fibroblast movement is directed by the orientation of the matrix, a phenomenon known as "contact guidance". This has been demonstrated directly when fibroblasts placed on oriented collagen gels invade in the direction of orientation (Guido \& Tranquillo, 1993) with further evidence provided by experiments demonstrating fi- 
broblast migration along fibronectin fibrils (Hsieh \& Chen, 1983). In the model we define the variable $\mathbf{v}^{i}(t)$ which represents the combined effect of the fibrin and collagen matrices on a cell's direction by

$$
\mathbf{v}^{i}(t)=(1-\alpha) \mathbf{c}\left(\mathbf{f}^{i}(t)\right)+\alpha \mathbf{b}\left(\mathbf{f}^{i}(t)\right)
$$

Unless otherwise stated we assume (in the absence of experimental data) that the fibrin and collagen have an equal effect and thus $\alpha=0.5$. In addition we define

$$
\mathbf{u}^{i}(t)=(1-\rho) \frac{\mathbf{v}^{i}(t)}{\left\|\mathbf{v}^{i}(t)\right\|}+\rho \frac{\mathbf{f}^{i}(t-\tau)^{\prime}}{\left\|\mathbf{f}^{i}(t-\tau)^{\prime}\right\|}
$$

which allows the cells to be polarized, a property we discuss later. The prime denotes differentiation with respect to time and $\tau$ is a time lag. The equation determining $\mathbf{f}^{i}$ is given by

$$
\mathbf{f}^{i}(t)^{\prime}=s\left(\left\|\mathbf{c}\left(\mathbf{f}^{i}(t)\right)\right\|,\left\|\mathbf{b}\left(\mathbf{f}^{i}(t)\right)\right\|\right) \frac{\mathbf{u}^{i}(t)}{\left\|\mathbf{u}^{i}(t)\right\|} .
$$

The extracellular matrix also alters fibroblast speed, an effect taken into account in equation 3. In particular, fibroblasts migrate more easily on fibronectin gels than on collagen gels (Wojciak-Stothard et al., 1992). Fibronectin is a protein found in abundance in the fibrin-based blood clot, where it coats the fibrin, promoting cell migration into the clot (Clark, 1996). Although the model formulation does not explicitly include fibronectin, we assume that it is proportional to the density of fibrin within the blood clot. We take the cell speed to be a function of collagen and fibronectin densities at the cell location, with the speed increasing with fibronectin density and conversely decreasing with collagen density. The precise functional forms we use are given in the Appendix.

Our model neglects several known ways in which the extracellular matrix influences the fibroblasts. For example, the collagen content of the cell substrate alters the production of different proteins by the fibroblast (Clark et al., 1995); the extracellular matrix in the wound region contains many growth factors and cytokines which alter fibroblast behaviour. We exclude these factors and focus on contact guidance and speed variation, because they directly affect the alignment of new collagen.

Fibroblast regulation of the extracellular matrix. The influence of the fibroblasts on the extracellular matrix can also be divided into two parts, the first of which 
is collagen alignment. Although collagen can polymerize into thin fibrils, the organization of these into the fibrous structure seen in the dermis requires cellular intervention (Ehrlich \& Krummel, 1996). Fibroblasts produce collagen in compartments which are formed by deep involutions in the cell membrane (Birk \& Trelstad, 1986). From this observation, it is theorized that even though the collagen matrix is formed in an extracellular space, the cell controls the alignment and structure via the microenvironment in the involution. We represent the "combined influence of nearby cells" as a weighted sum of the cell direction defined by

$$
\mathbf{f}(\mathbf{x}, t)=\sum_{i=0}^{M} w\left(\mathbf{f}^{i}(t)-\mathbf{x}\right) \frac{\mathbf{f}^{i}(t-\tau)^{\prime}}{\left\|\mathbf{f}^{i}(t-\tau)^{\prime}\right\|},
$$

with the weights designed to restrict the influence to within about $10 \mu \mathrm{m}$ (see the appendix). We assume that the cells orient or reorient the collagen matrix towards their direction of motion. This is modelled by an ordinary differential equation for the rate of change of the angle of collagen orientation given by

$$
\frac{d \theta(t)}{d t}=\kappa\|\mathbf{f}\| \sin (\phi-\theta)
$$

where $\theta$ is the direction of the collagen matrix at a point in space, $\kappa$ is a parameter which measures the ability of the fibroblasts to reorder the matrix and $\phi$ is the direction of the vector $\mathbf{f}$. The angle changes most when the orientation of the collagen and the combined influence of the nearby cells differs by 90 degrees, and does not change at all if they are the same. We also incorporate a time lag in the cell direction to represent the delay between the orientation of the cell and the actual cell motion.

The second way the fibroblasts influence the extracellular matrix is by altering the density of the two types of protein. We assume that each fibroblast produces collagen at a constant rate and degrades both collagen and fibrin; there is no production of fibrin, which is deposited by the blood plasma when the fibrin clot is formed and enters the model as initial conditions. Thus the equations for the protein densities are given by

$$
\begin{aligned}
\frac{d\|\mathbf{c}\|}{d t} & =\left(p_{c}-d_{c}\|\mathbf{c}\|\right) \sum_{i=1}^{M} w\left(\mathbf{f}^{i}-\mathbf{x}\right) \\
\frac{d\|\mathbf{b}\|}{d t} & =-d_{b}\|\mathbf{b}\| \sum_{i=1}^{M} w\left(\mathbf{f}^{i}-\mathbf{x}\right)
\end{aligned}
$$

where $p_{c}, d_{c}$ and $d_{b}$ are positive constants. 
Other properties of the fibroblasts. The final elements of the model deal with properties of the fibroblasts: cell polarization and cell division. In addition to the contact guidance, cell direction is also affected by the morphology of the cell. When placed on fibrous gels, fibroblasts become highly elongated (Dunn \& Ebendal, 1978) and are polarized, with clear leading and trailing edges. We model polarization by assuming that the cells tend to maintain their current direction of motion (see equation 2). The overall fibroblast direction is thus given by a weighted vector sum of the contact guidance direction and the current direction (equation 3).

The other property that we incorporate into the model is cell division. In doing this, we allow for two cell types, those which divide and those which do not. The division rate is taken to be constant throughout the simulation, with dividing fibroblasts becoming non-dividing. Because of the discrete nature of the model, it is necessary to explicitly separate the daughter cells by a small distance. This is done by placing the daughter cell at a randomly chosen point on the circle centered at the parent's location with a specified radius (taken to be $10 \mu \mathrm{m}$ ).

Most of the parameters in the model represent quantitative details of fibroblast behaviour, which are not well known. This means that it is important to determine how sensitive the results of the model are to the parameters, which is the goal of section 3. A list of typical parameter values is given in the Appendix.

\section{Variations in Model Parameters}

Having given a description of the model in the previous section, we now turn our attention to numerical investigations of model behaviour. Figure 1 shows the results of a typical simulation for tissue regeneration in a rodent slash wound. The domain is a two dimensional cross section of the wound transverse to the skin surface. Throughout the remainder of the paper we treat this as a standard case with which the other simulations can be compared. Figure 1 (a) and (c) demonstrate typical features of scarring, in particular a denser collagen region with more pronounced alignment close to the subcutaneous region; the collagen becomes less organized closer to the skin surface. These are natural consequences of the fact that 80 percent of the fibroblasts which enter the wound from the surrounding tissue do so from the subcutaneous fascia (Adams, 1997). When the cells enter the wound region they encounter a randomly 

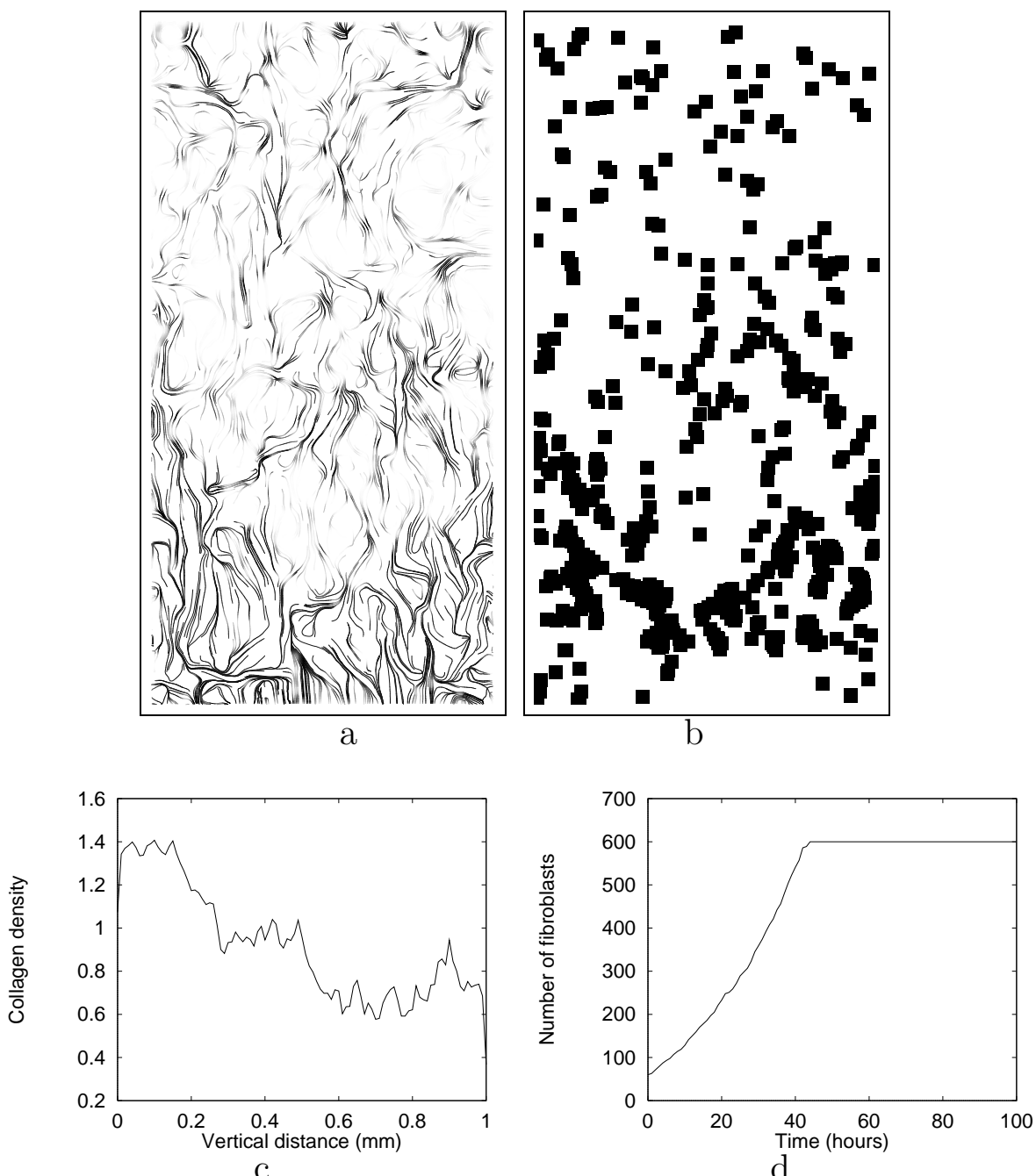

Figure 1. Simulation results for a typical rodent slash wound (1 $\mathrm{mm}$ deep and $0.5 \mathrm{~mm}$ wide) approximately four days post wounding. In (a) the collagen alignment and density are shown in a wound region with the right and left boundaries representing interfaces with normal dermis, the bottom representing the interface with the subcutaneous fascia and the top representing the interface with the epidermis. The wound region with the location of the fibroblasts at the end of the simulation is shown in (b). The black squares marking the cells' locations also denote the region of extracellular matrix that the cells modify or the support of the weight function. The collagen density integrated over the horizontal direction is shown in (c). The vertical distance is measured from the fascia. In (d) the number of fibroblasts in the wound region is plotted against time. The simulation runs for 100 hours where $t=0$ would represent somewhere between 24 and 48 hours postwounding when the fibroblasts start to enter the wound region. The alignment is shown by drawing lines whose tangent corresponds to the collagen vector field (the streamlines) with black representing high collagen density and white representing low collagen density. The parameters used are given in the Appendix. The cells deflect off the boundary by reflecting the component of motion perpendicular to the boundary. 
aligned fibrin clot which is gradually degraded and replaced with collagen fibres. As the simulation progresses, cells in the wound region can divide and other cells can continue moving in from the edges, increasing the cell density until there is a maximum of 600 cells as shown in figure 1(d). High cell density causes more collagen production and fibrin degradation which slows the cells, and likewise high collagen density causes more cell accumulation as the cells slow down and continue to divide. This self reinforcing feature can be seen in figure 1(b). We now consider the ways in which the collagen alignment is affected by varying key parameters in the model.

Influx from surrounding tissue verses cell multiplication. The first parameter we vary is the cell cycle time. Due to the design of the model, this affects the way the cells enter the wound region. Recall that in the model the cells are introduced into the domain from adjacent dermis and the subcutaneous fascia at a constant rate, and divide until a maximum number of cells in the wound region is reached. Thus by increasing the cell cycle time, a smaller proportion of cells enter via multiplication relative to migration. Similarly, by decreasing the cell cycle time the situation is reversed, with more cell divisions occurring. Changes in how the cells enter the wound region have a dramatic effect on the alignment and density patterns, as shown in figure 2. In (a), all the cells enter the wound from the surrounding tissue ( 80 percent from the fascia) and in part (b), 60 cells enter initially from surrounding tissue with all the rest resulting from cell division. Both the alignment and density show dramatic differences. In (a) there is a dense and very aligned region of collagen at the base of the wound area, as is commonly seen in experiments, while in (b) the collagen is less aligned, with a dense region away from the base and extending up into the centre of the wound region. This suggests that fibroblasts entering the wound from surrounding tissue play a more important role in scar tissue formation than those which are introduced into the wound region by cell division.

Variation in cell numbers. Having concluded that the way in which fibroblasts enter the wound region is important in collagen alignment, we next investigate how changes in the maximum number of cells influence the alignment. The model allows for a maximum number of cells to exist in the $0.5 \mathrm{~mm} \times 1 \mathrm{~mm}$ wound region which, for our standard case, corresponding to experimental results, is 600 cells (Adams, 1997). By increasing this maximum, the collagen is more quickly deposited and there is increased 

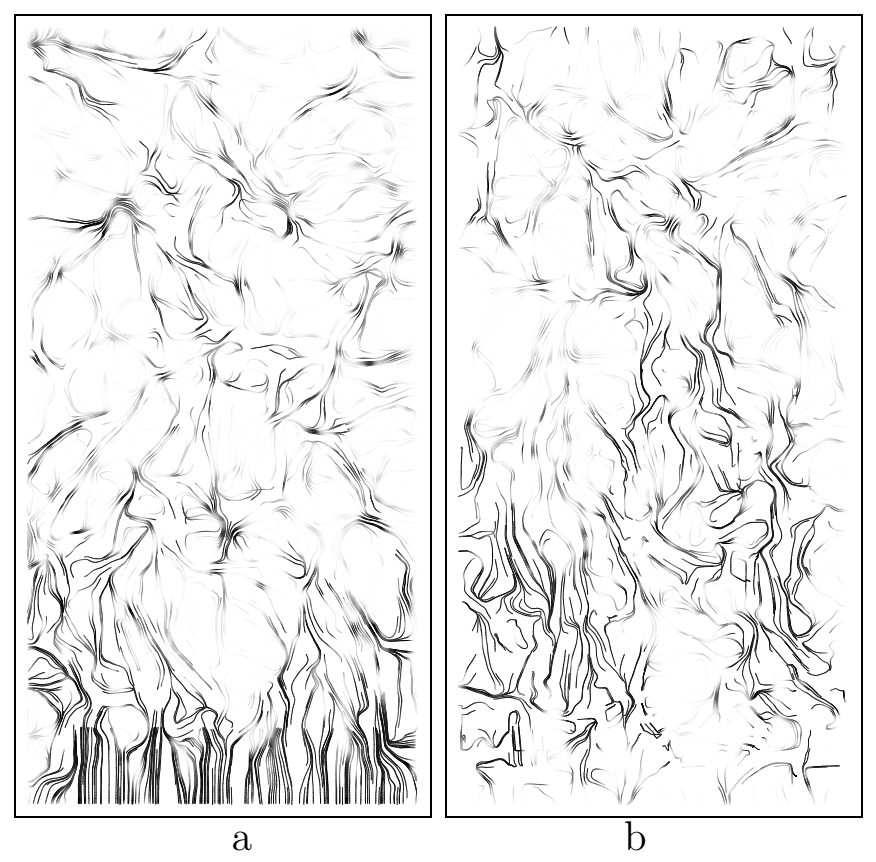

Figure 2. Collagen alignment and density for a simulation in which all cells enter the wound from the surrounding tissue (a) with 80 percent coming from the fascia. In (b), 60 cells are initially placed at the wound periphery (48 at the base) with subsequent cells arising via cell division. The strong alignment at the base of (a) is due to most of the cells entering from that region. Comparing (a), (b) and figure 1(a), the strong effect of cell flux can be seen. The alignment is shown by drawing lines whose tangent corresponds to the collagen vector field (the streamlines) with black representing high collagen density and white representing low collagen density. Model details are as in figure 1. 

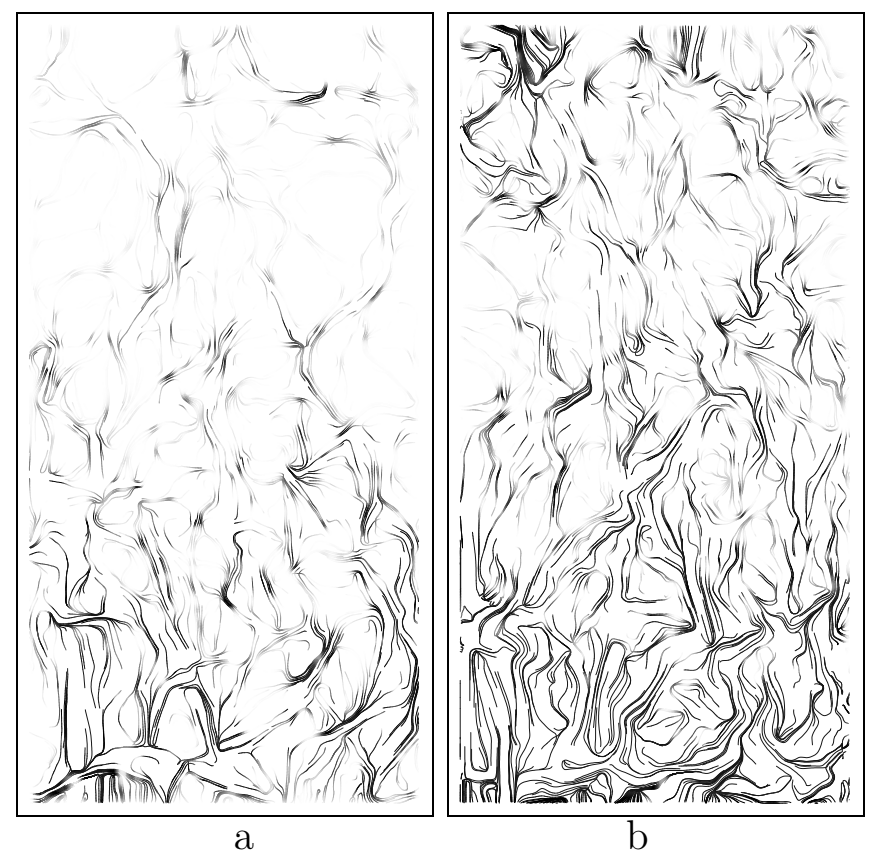

Figure 3. Collagen alignment and density for simulations which differ from figure 1 in the total number of cells. In (a) there is a maximum of 200 fibroblasts and in (b) there is a maximum of 1200 fibroblasts. The lower collagen density in (a) is due to fewer fibroblasts in the simulation. The orientation in (b) of the collagen is more aligned than in (a) but not significantly more than in figure 1(a). This indicates that total cell number is not significant in aligning the collagen. The alignment is shown by drawing lines whose tangent corresponds to the collagen vector field (the streamlines) with black representing high collagen density and white representing low collagen density.

alignment because more cells enter from the subcutaneous fascia. The cause of alignment is verified by reducing the influx from the fascia, while maintaining the higher cell maximum, which results in a more random pattern. When the maximum number of cells is decreased, there is less collagen produced and the alignment is reduced slightly due to fewer cells entering from the fascia.

Variations in cell speed. The nature of the collagen alignment is strongly determined by the speed of the fibroblasts. In figure 4(a) and figure 4(b) the results of two simulations are shown where the cell speed is changed so that the average cell speed is first lower and then higher than the standard case (figure 1). The simulation with low cell speed was run for a longer time (300 hours) so that the degree of invasion is comparable with the standard case. It is clearly seen that with faster cell speeds there is more alignment than with lower speeds. This is due to our assumption that higher speeds cause the cells to move for longer runs before sampling their environment to 


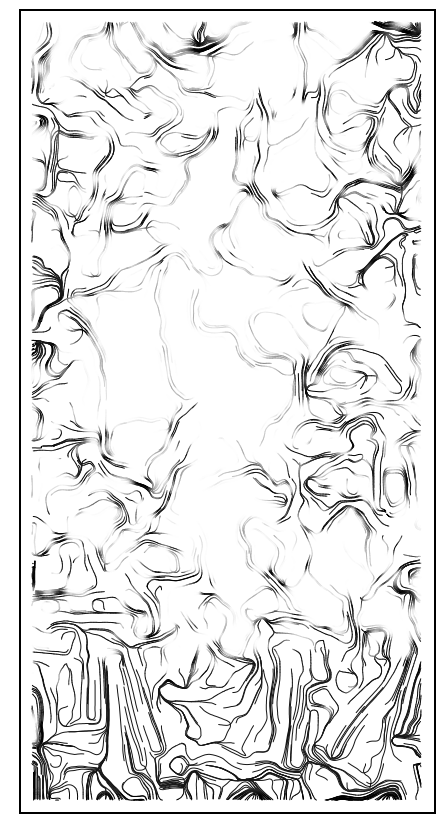

a: Low speed

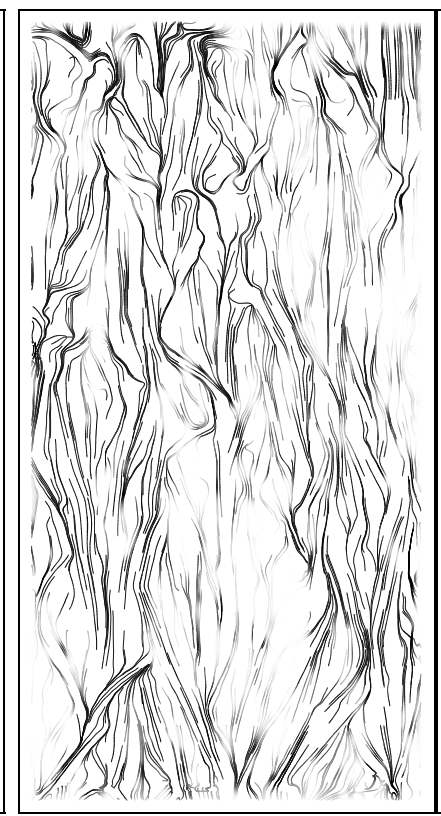

b: High speed

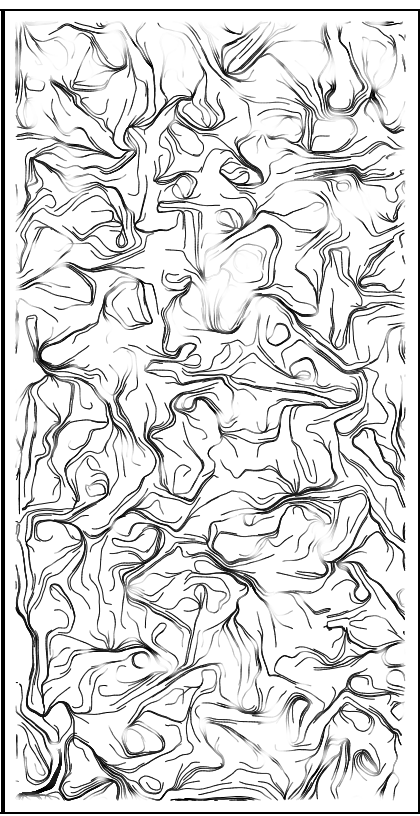

c: Blocked fascia

Figure 4. Collagen alignment and density for simulations where the fibroblasts' speeds are lower than normal (a) and higher than normal (b). In (c) a more random collagen alignment pattern develops in a simulation where the influx of cells from the subcutaneous fascia is blocked but the cells' speeds are normal. The significant alignment in (b) when compared to (a) shows the importance of speed in aligning the collagen. In (a), $\nu=5 \mu \mathrm{m}$ per hour giving an average cell speed at $t=100$ of $0.747 \mu \mathrm{m}$ per hour and in (b) $\nu=30 \mu \mathrm{m}$ per hour giving an average cell speed at $t=100$ of $7.62 \mu \mathrm{m}$ per hour. This can be compared with figure 1 where $\nu=30 \mu \mathrm{m}$ per hour with an average cell speed of $3.16 \mu \mathrm{m}$ per hour. The collagen in (a) is shown at $t=300$ hours,in (b) at $t=100$ and in (c) at $t=200$. The alignment is shown by drawing lines whose tangent corresponds to the collagen vector field (the streamlines) with black representing high collagen density and white representing low collagen density. The model parameters are the same as those in figure 1 unless otherwise noted.

determine a new direction.

\section{Model Predictions for Experimental Interventions}

In the previous section we investigated the model by examining the effects of changing various parameters. With a more fundamental understanding of the model, we now describe the results from three simulations representing potential biological experiments whose results would shed light on the healing process.

- In the first simulated experiment, cells entering the wound directly from the subcutaneous fascia are blocked (figure 4c). Comparison with figure 1 shows that the collagen is not as strongly aligned in the vertical direction when the cells are 
blocked resulting in less scarring, but initial collagen deposition is slower (due to the lower cell densities). In a simulation with roughly equal numbers of cells entering from each wound edge, collagen alignment patterns very similar to figure 4(c) result, without the slower collagen buildup. When 80 percent of the fibroblasts enter at the fascia, vertical alignment is obtained; this supports the conclusion of the previous section that the manner in which the cells are introduced into the wound region is key in establishing alignment. The key difference in the blocked fascia simulation when compared with the standard case, is the flux relative to the density of dividing cells. Our model assumes that cell division disrupts fibroblast polarization and thus has a randomizing influence on the alignment pattern. A lower flux of cells from the surrounding tissue allows more cell proliferation, causing less alignment.

- Our model predicts that enhancing cell division (for example, by addition of a chemical mitogen throughout the wound and surrounding tissue) should cause less scarring. Decreasing the cell cycle time has a slight randomizing effect on collagen alignment due to the loss of cell polarization that results from cell division. However, a more important effect is how the cell population in the wound region responds. If, as a result of more cells dividing, fewer cells migrate into the region, there will be less alignment. By suppressing cell division, the most important effect is again on the cell density. There will be a strong tendency to alignment if there is an increased cell flux; otherwise, the cell densities will be lower, the cell speeds will be faster and the tendency will again be to align. In the wound region, platelet-derived growth factor acts as a chemoattractant for fibroblasts, as well as promoting cell proliferation (Heldin \& Westermark, 1996). Our model suggests that these are two factors which oppose each other in terms of aligning the collagen matrix, suggesting that this cytokine is not a key regulator of alignment.

- Altering the rates of production and degradation of collagen (which could be achieved, at least in vitro, by gene amplification) will obviously alter its density in the wound region. Our model predicts that there is also an effect on collagen alignment, due to the dependency of the cell speed on the protein densities. By altering the production and degradation terms, the local densities are changed, 
leading to altered speeds of fibroblast movement, and thus causing changes in alignment. If the collagen deposition is slower the average cell speed is faster causing greater alignment and conversely if the collagen deposition is faster the average cell speed is slower causing less alignment.

\section{Discussion}

Our model has indicated various phenomena at the cellular level that are important in collagen alignment during wound repair. We begin the discussion by considering how these relate to the new antiscarring therapies currently being developed. Neutralizing antibody to TGF $\beta$ has been suggested as a novel anti-scarring agent (Shah et al., 1992). More recently, it has been found that manipulating the concentrations of different isoforms of TGF $\beta$ has different effects (Shah et al., 1995). These isoforms influence all phases of the healing process, including complicated modifications to fibroblast behaviour. At least two effects on the fibroblasts are pertinent to this discussion. First, TGF $\beta_{1}$ induces fibroblasts to produce more collagen in fibrin gels and to a lesser degree in collagen gels (Clark et al., 1995). Second, although TGF $\beta_{1}$ act as a chemoattractant for fibroblasts, it can also inhibit migration into three-dimensional collagen gels (Roberts \& Sporn, 1996). In the context of our model, altering the collagen production does not directly influence the manner in which collagen is aligned, but it changes cell speed which affects alignment. Because TGF $\beta$ can act as a chemoattractant, our model predicts two possible causes for different alignment: modified cell flux into the wound region and changes in the speed of the fibroblasts. Experimental results show that neutralizing $\operatorname{TGF} \beta_{1}$ results in the initial collagen deposition being decreased with only slightly improved scarring, whereas neutralizing both $\operatorname{TGF} \beta_{1}$ and $\operatorname{TGF} \beta_{2}$ or adding $\operatorname{TGF} \beta_{3}$ causes less scarring. This is consistent with our model's predictions, indicating that the primary reason for reduced scarring is not a direct result of the slower deposition of collagen (see Section 4). If neutralizing both $\operatorname{TGF} \beta_{1}$ and $\operatorname{TGF} \beta_{2}$ reduces the influx of cells from the fascia, our model predicts the observed results of less scarring and slower initial collagen deposition.

Thus, even in the extremely simplified caricature of tissue regeneration represented by our model, collagen alignment is clearly shown to be a multi-factorial process. This is consistent with experimental evidence, and implies that therapeutic intervention must 
address several separate causes of the altered alignment pattern. Most randomizing processes in the system, such as the random structure of the fibrin clot will, over time, tend to disrupt alignment in the absence of factors which tend to cause alignment. These factors include cell speed, flux, division and other secondary effects which influence these, such as collagen production rate. The most important alignment-producing features are cell speed and cell flux. By moving faster, our model fibroblasts less frequently receive contact guidance cues, causing them to travel in straighter paths and give the collagen greater alignment. However, a more important feature for scar formation is the manner in which the cells enter the wound region. Our model predicts that the fundamental reason for the structural differences between scar and normal tissue is not the transition between fibrin and collagen matrices, or biochemical differences in the wound region, but simply the fact that fibroblasts establish an alignment pattern as they migrate into the wound from the periphery. This is highlighted by the results of a simulation where the maximum number of cells are uniformly placed in the fibrin clot rather than dividing or migrating in from the wound edges. This results in collagen patterns which are similar to that shown in figure 4(c), with randomly aligned collagen, more closely resembling the situation in normal skin. As the fibroblasts flow into a wound from the surrounding tissue (predominantly from the subcutaneous tissue), they generate collagen fiber patterns which are reinforced by subsequent fibroblast migration. This is the basic process by which the matrix becomes aligned and scar tissue forms.

In our modelling we have focused on the initial deposition of collagen. We believe that this is the most important factor in determining alignment due to self reinforcing processes. Yet there are several elements which could be considered in future modelling work. Wound contraction is an important process in wound healing and could reinforce initial collagen alignment. The chemical regulation of the wound environment by growth factors is also a key regulator of fibroblasts during wound repair. These are two elements which could have significant consequences for collagen alignment and there are many others which would provide a more complete picture of wound healing.

Acknowledgements. This research was supported by EPSRC grant GR/K71394 and London Mathematical Society, under scheme 3. We also acknowledge support from the Institute for Mathematics and its Applications (University of Minnesota) with funds 
provided by the National Science Foundation

\section{Appendix}

Here we describe specific functions and parameters used for the standard simulation. Our description is deliberately brief and further mathematical details are published elsewhere (Dallon et al., 1999; Dallon, 2000).

\section{Speed Function}

The dependency of the speed on the protein densities is given by

$$
s=\nu\left(e_{1}+\frac{e_{2}}{1+e_{3}\|\mathbf{c}\|^{q}}\right)\left(\frac{1+g_{1}\|\mathbf{b}\|}{g_{2}}\right)
$$

where $\nu, e_{1}, e_{2}, e_{3}, q, g_{1}$ and $g_{2}$ are a positive constant. The function is chosen so that the first product is a decreasing function ranging from 1 at $\|c\|=0$ to about 0.208 at $\|c\|=1.5$, and the second product is a linearly increasing function ranging from 0.33 to 1 for the values of $\|b\|$. Thus the cell speed has a maximum value of $\nu$ and a minimum value of about $\nu / 9$. The constants used in the simulations are $e_{1}=1 / 8, e_{2}=7 / 8$, $e_{3}=3 / 8, q=6, g_{1}=2, g_{2}=3$ and $\nu=30$ unless stated otherwise.

\section{Equation for the Weight Function}

The weight function from equation 4 is defined by

$$
w\left(\mathbf{f}^{i}-\mathbf{x}\right)=a_{1} a_{2} \text { with } a_{j}=\max \left\{1-\frac{\left|f_{j}^{i}-x_{j}\right|}{L}, 0\right\},
$$

where $\mathbf{f}^{i}=\left(f_{1}^{i}, f_{2}^{i}\right), \mathbf{x}=\left(x_{1}, x_{2}\right)$ and $L=10 \mu \mathrm{m}$. Thus a cell will influence the extracellular matrix which is in a $20 \mu \mathrm{m}$ square centered at the cell.

\section{The Numerical Method}

The equations are solved by discretizing the variables $\mathbf{c}$ and $\mathbf{b}$ in space and then solving the ordinary differential equations at the appropriate grid points using Euler's method. The cells are not constrained to the discretized grid and thus the grid variables must be interpolated to the cell locations and the cell influence must be interpolated to the grid locations. The first interpolation uses a standard tensor product piecewise 
quartic interpolant. For the second interpolation we use a discretized version of $w$. The numerical algorithm used to solve these equations consists of the following steps:

(i) Interpolate the magnitude of the collagen and fibrin as well as the direction of the collagen to the fibroblast locations.

(ii) Interpolate the influence of the fibroblasts to the extracellular matrix.

(iii) Determine the fibroblasts direction (equation 3) using an explicit Euler method.

(iv) Solve the direction and magnitude of the collagen and the magnitude of the fibrin (equations 5, 6 and 7) on a fixed grid using an explicit Euler method.

(v) Move the fibroblasts to their new locations and decide if they should divide.

\section{Parameter values}

The domain is taken to be $0.5 \mathrm{~mm}$ by $1 \mathrm{~mm}$ with the maximum number of fibroblasts $M=600$, values which compare well with typical rodent slash wounds. The discretization used for the domain is a grid of 51 by 101 points and a time step of 0.15 hours. The cell cycle time is 18 hours and the base cell speed $\nu=15.0 \mu \mathrm{m}$ per hour. These are standard values for fibroblasts. The other parameter values represent qualities of the fibroblasts and are difficult to determine or have not been measured experimentally. The time lag $\tau=0.15$ (9 minutes) hours was chosen since the cell moves at most 2.25 microns in this time interval and for computational convenience. A range of values for $\kappa$, which represents the effectiveness of the fibroblasts in reorienting the collagen, from 0 to 40 were tested in experiments. The value used for the simulations in this paper is $\kappa=5.0$. The cell polarization parameter does not dramatically affect the collagen alignment (see Section 3) and in all the simulations shown we take $\rho=0.9$, reflecting the high degree of polarity in fibroblasts on fibrous gels. The value for $\alpha$ is chosen to be 0.5 assuming that the fibrin clot and the collagen matrix would equally influence the direction of the fibroblasts. Simulations show that our results are not sensitive to changes in this parameter. The production of collagen by fibroblasts is well researched, but only in terms of ratios with respect to normal production. Typically the protein is radioactively labelled and the results are measured in increments of several days. This essentially gives at most two data points for the time interval which we are examining. The values we use for the production and degradation of collagen and fibrin are 
$p_{c}=0.64, d_{c}=0.44$ and $d_{b}=0.6$, which are rough estimates giving an appropriate time course for the production and degradation of the proteins.

\section{References}

Adams, J. J. 1997 The cell kinetics of murine incisional wound healing Ph.D. thesis, University of Manchester.

Anderson, A. R. A., \& Chaplain, M. A. J. 1998 Continuous and Discrete Mathematical Models of Tumor-induced Angiogenesis. Bull. Math. Biol., 60(5), 857-899.

Anderson, A. R. A., Chaplain, M. A. J., Newman, E. L., Steele, R. J. C., \& Thompson, A. M. 2000 Mathematical Modelling of tumour invasion and metastasis. J. Theor. Med., 2, 129-154.

Armstrong, J. R., \& Ferguson, M. W. 1995 Ontogeny of the Skin and the Transition from Scar-Free to Scarring Phenotype during Wound Healing in the Pouch Young of a Marsupial, Monodelphis domestica. Dev. Biol., 169, 242-260.

Birk, D. E., \& Trelstad, R. L. 1986 Extracellular Compartments in Tendon Morphogenesis: Collagen, Fibril, Bundle, and Macroaggregate Formation. J. Cell Biol., 103, 231-240.

Clark, R. A. F. 1996 Wound Repair Overview and General Considerations. Pages 3-50 of: Clark, R. A. F. (ed), The Molecular and Cellular Biology of Wound Repair, 2 edn. Plenum Press.

Clark, R. A. F., Nielsen, L. D., Welch, M. P., \& McPherson, J. M. 1995 Collagen matrices attenuate the collagen-synthetic response of cultured fibroblasts to TGF3. J. Cell Sci., 108, 1251-1261.

Dale, P. D., Sherratt, J. A., \& Maini, P. K. 1996 A mathematical model for collagen fibre formation during foetal and adult dermal wound healing. Proc. R. Soc. Lond. $B, \mathbf{2 6 3}, 653-660$.

Dallon, J. C. 2000 Numerical Aspects of Discrete and Continuum Hybrid Models in Cell Biology. Applied Numerical Mathematics, 32, 137-159.

Dallon, J. C., Sherratt, J. A., \& Maini, P. K. 1999 Mathematical Modelling of Ex- 
tracellular Matrix Dynamics using Discrete Cells: Fiber Orientation and Tissue Regeneration. J. Theor. Biol., 199(4), 449-471.

Dunn, G. A., \& Ebendal, T. 1978 Contact guidance on oriented collagen gels. Exp. Cell Res., 111, 475-479.

Edelstein-Keshet, L., \& Ermentrout, B. G. 1990 Models for contact-mediated pattern formation: cells that form parallel arrays. J. Math. Biol., 29, 33-58.

Ehrlich, P. H., \& Krummel, T. M. 1996 Regulation of wound healing from a connective tissue perspective. Wound Rep. Reg., 4(April-June), 203-210.

Forrest, L. 1983 Current concepts in soft connective tissue wound healing. Br. J. Surg., 70, 133-140.

Guido, S., \& Tranquillo, R. T. 1993 A methodology for the systematic and quantitative study of cell contact guidance in oriented collagen gels. J. Cell Sci., 105, 317-331.

Hay, E. D. (ed). 1991 Cell Biology of Extacellular matrix. 2 edn. Plenum Press.

Heldin, C.-H., \& Westermark, B. 1996 Role of Platelet-Derived Growth Factor in Vivo. Pages 249-273 of: Clark, R. A. F. (ed), The Molecular and Cellular Biology of Wound Repair, 2 edn. Plenum Press.

Hsieh, P., \& Chen, L. B. 1983 Behavior of cells seeded on isolated fibronectin matrices. J. Cell Biol., 96, 1208-1217.

Jennings, R. W., \& Hunt, T. K. 1992 Fetal Wound Healing. Elsevier Science Publishing Company.

McCallion, R. L., \& Ferguson, M. W. J. 1996 Fetal Wound Healing and the Development of Antiscarring Therapies for Adult Wound Healing. Pages 561-600 of: Clark, R. A. F. (ed), The Molecular and Cellular Biology of Wound Repair, 2 edn. Plenum Press.

Olsen, L., Sherratt, J. A., \& Maini, P. K. 1995 A Mechanochemical Model for Adult Dermal Wound Contraction and the Permanence of the Contracted Tissue Displacement Profile. J. Theor. Biol., 177, 113-128.

Olsen, L., Maini, P. K., Sherratt, J. A., \& Dallon, J. C. 1999 Mathematical Modelling of Anisotropy in Fibrous Connective Tissue. Math. Biosci., 158, 145-170. 
Roberts, A. B., \& Sporn, M. B. 1996 Transforming Growth Factor- $\beta$. Pages 275-308 of: Clark, R. A. F. (ed), The Molecular and Cellular Biology of Wound Repair, 2 edn. Plenum Press.

Shah, M., Foreman, D. M., \& Ferguson, M. W. J. 1992 Control of scarring in adult wounds by neutralising antibody to transforming growth factor $\beta$. Lancet, 339, $213-214$.

Shah, M., Foreman, D. M., \& Ferguson, M. W. J. 1995 Neutralisation of TGF- $\beta_{1}$ and TGF- $\beta_{2}$ or exogenous addition of TGF- $\beta_{3}$ to cutaneous rat wounds reduce scarring. J. Cell Sci., 108, 985-1002.

Stott, E. L., Britton, N. F., Glazier, J. A., \& Zajac, M. 1999 Stochastic simulation of benign avascular tumour growth using the Potts model. Math. Comp. Modelling, 30, 183-198.

Tracqui, P., Woodward, D. E., Cruywagen, G. C., Cook, J., \& Murray, J. D. 1995 A mechanical model for fibroblast-driven wound healing. J. Biol. Systems, 3, 10751084.

Tranquillo, R. T., \& Murray, J. D. 1992 Continuum model of fibroblast-driven wound contraction: Inflammation-mediation. J. Theor. Biol., 158, 135-172.

Whitby, D. J., \& Ferguson, M. W. J. 1991 The extracellular matrix of lip wounds in fetal, neonatal and adult mice. Dev., 112, 651-668.

Wojciak-Stothard, B., Denyer, M., Mishra, M., \& Brown, R. A. 1992 Adhesion orientation, and movement of cells cultured on ultrathin fibronectin fibers. In Vitro Cell. Devel. Biol., 33(2), 110-117. 